\title{
SHARPNESS OF UNIFORM CONTINUITY OF QUASICONFORMAL MAPPINGS ONTO $s$-JOHN DOMAINS
}

\author{
Chang-Yu Guo and Pekka Koskela
}

\author{
University of Jyväskylä, Department of Mathematics and Statistics \\ P. O. Box 35, FI-40014 University of Jyväskylä, Finland; changyu.c.guo@jyu.fi \\ University of Jyväskylä, Department of Mathematics and Statistics \\ P. O. Box 35, FI-40014 University of Jyväskylä, Finland; pkoskela@maths.jyu.fi
}

\begin{abstract}
We show that a prediction in [8] is inaccurate by constructing quasiconformal mappings onto $s$-John domains so that the mappings fail to be uniformly continuous between natural distances. These examples also exhibit the sharpeness of the assumptions in [5].
\end{abstract}

\section{Introduction}

Recall that a conformal mapping of the unit disk onto a bounded domain $\Omega$ is uniformly $\alpha$-Hölder continuous, $0<\alpha \leq 1$, if and only if the hyperbolic metric $\rho_{\Omega}$ in $\Omega$ satisfies the logarithmic growth condition

$$
\rho_{\Omega}\left(z_{0}, z\right) \leq \frac{1}{\alpha} \log \frac{\operatorname{dist}\left(z_{0}, \partial \Omega\right)}{\operatorname{dist}(z, \partial \Omega)}+C_{0},
$$

where $z_{0}=f(0)$ and $C_{0}<\infty$. Here dist refers to the Euclidean distance. This result is due to Becker and Pommerenke [2].

Gehring and Martio [3] gave a quasiconformal analogue of the result be Becker and Pommerenke by replacing the hyperbolic metric in (1.1) with the quasihyperbolic metric. Recall that the quasihyperbolic distance between $x$ and $x_{0}$ in $\Omega \neq \mathbf{R}^{n}$ is

$$
k_{\Omega}\left(x, x_{0}\right)=\inf _{\gamma_{x}} \int_{\gamma_{x}} \frac{d s}{\operatorname{dist}(z, \partial \Omega)},
$$

where the infimum is taken over all rectifiable curves $\gamma_{x}$ in $\Omega$ which join $x$ to $x_{0}$. For $x, y \in \Omega$, there is a (quasihyperbolic) geodesic $[x, y]$ in $\Omega$ with

$$
k_{\Omega}(x, y)=\int_{[x, y]} \frac{d s}{\operatorname{dist}(z, \partial \Omega)},
$$

see [4]. In particular, they showed that the condition

$$
k_{\Omega}\left(z_{0}, z\right) \leq \frac{1}{\alpha} \log \frac{\operatorname{dist}\left(z_{0}, \partial \Omega\right)}{\operatorname{dist}(z, \partial \Omega)}+C_{0},
$$

guarantees that, given $\Omega^{\prime}$ and a $K$-quasiconformal mapping $f: \Omega^{\prime} \rightarrow \Omega$, the restriction of $f$ to any ball $B \subset \Omega^{\prime}$ is uniformly Hölder continuous with an exponent $\beta$ and a constant $M$ that both are independent of $B$. Under suitable geometric conditions on $\Omega^{\prime}$ they then concluded uniform Hölder continuity in the entire $\Omega^{\prime}$.

https://doi.org/10.5186/aasfm.2017.4202

2010 Mathematics Subject Classification: Primary 30C62, 30C65.

Key words: $s$-John domain, uniform continuity, quasiconformal mapping, internal diameter, internal metric.

C.-Y. Guo and P. Koskela were partially supported by the Academy of Finland via the Centre of Excellence in Analysis and Dynamics Research (project No. 271983). 
In [10], Koskela, Onninen and Tyson showed that (1.2) actually implies that a quasiconformal mapping $f: \Omega^{\prime} \rightarrow \Omega$ is always uniformly Hölder continuous when $\Omega^{\prime}$ is equipped with the internal distance $d_{I}(z, w)$ and $\Omega$ with the usual Euclidean distance. Recall that $d_{I}(z, w)$, for a pair of points in a domain $G$, is the infimum of the lengths of all paths that join $z$ to $w$ in $G$.

In [8], Hencl and Koskela relaxed (1.2) to

$$
k_{\Omega}\left(x, x_{0}\right) \leq \phi\left(\frac{1}{\operatorname{dist}(x, \partial \Omega)}\right)
$$

under the assumption that

$$
\int_{1}^{\infty} \frac{d t}{\phi^{-1}(t)}<\infty
$$

A uniform continuity estimate with respect to the internal metric in $\Omega^{\prime}$ and the Euclidean metric in $\Omega$ was established under the additional assumption that $t \mapsto$ $\Phi(t)^{-a}$ is concave for some $a>n-1$, where

$$
\Phi(t)=\psi^{-1}(t) \quad \text { and } \quad \psi(t)=\int_{t}^{\infty} \frac{d s}{\phi^{-1}(s)} .
$$

This concavity assumption was speculated in [8] to be superfluous.

Let us recall a class of domains for which growth conditions of the above type are easily verified. First of all, a bounded domain $\Omega \subset \mathbf{R}^{n}$ is a John domain if there is a constant $C$ and a point $x_{0} \in \Omega$ so that, for each $x \in \Omega$, one can find a rectifiable curve $\gamma:[0,1] \rightarrow \Omega$ with $\gamma(0)=x, \gamma(1)=x_{0}$ and with

$$
C \operatorname{dist}(\gamma(t), \partial \Omega) \geq l(\gamma([0, t]))
$$

for each $0<t \leq 1$. John used this condition in his work on elasticity [9] and the term was coined by Martio and Sarvas [11]. Smith and Stegenga [13] introduced the more general concept of an $s$-John domain, $s \geq 1$, by replacing (1.5) with

$$
C d(\gamma(t), \partial \Omega) \geq l(\gamma([0, t]))^{s} .
$$

The recent studies $[1,6,7]$ on mappings of finite distortion have generated new interest in the class of $s$-John domains. Direct integration along a curve from the definition of a John domain gives (1.2) with $z_{0}$ replaced by $x_{0}$ for the 1-John case and (1.3) with $\phi(t)=C t^{s-1}$ in the case of an $s$-John domain, $s>1$. It is easy to check that the concavity assumption on the associated $\Phi$ holds if $s<1+\frac{1}{n}$ and that the convergence condition holds when $s<2$. On the other hand, Guo has established in [5] the above uniform continuity result for $s$-John domains with $1 \leq s<1+\frac{1}{n-1}$. Our first result shows that the concavity assumption is not superfluous and that the requirement that $1 \leq s<1+\frac{1}{n-1}$ cannot be relaxed in the planar case.

Theorem 1.1. There exist a bounded 2-John domain $\Omega \subset \mathbf{R}^{2}$, a constant $C<$ $\infty$ and a point $x_{0} \in \Omega$ with

$$
k_{\Omega}\left(x, x_{0}\right) \leq C \operatorname{dist}(x, \partial \Omega)^{-\frac{1}{2}}
$$

for all $x \in \Omega$ so that a quasiconformal mapping $f: \Omega^{\prime} \rightarrow \Omega$ fails to be uniformly continuous with respect to the Euclidean metric in $\Omega$ and $d_{I}$ in $\Omega^{\prime}$ for a bounded domain $\Omega^{\prime} \subset \mathbf{R}^{2}$.

The above example is somewhat surprising since the modulus of continuity from [8] does not degenerate when the exponent $-a$ in $k_{\Omega}\left(x, x_{0}\right) \leq C \operatorname{dist}(x, \partial \Omega)^{-a}$ tends 
$-1 / n$ nor does the modulus of continuity in [5] when $s$ tends to $1+\frac{1}{n-1}$ in the $s$-John condition.

Our second result shows that the concavity condition is necessary in all dimensions and that the value $1+\frac{1}{n-1}$ is critical in the $s$-John condition.

Theorem 1.2. For each $n \geq 3$, there exist a bounded domain $\Omega^{\prime} \subset \mathbf{R}^{n}$ and a domain $\Omega \subset \mathbf{R}^{n}$ that is $s$-John for all $s>1+\frac{1}{n-1}$ with

$$
k_{\Omega}\left(x, x_{0}\right) \leq C \operatorname{dist}(x, \partial \Omega)^{-\frac{1}{n}} \log \frac{C}{\operatorname{dist}(x, \partial \Omega)}
$$

for some constant $C<\infty$, all $x \in \Omega$ and a quasiconformal mapping $f: \Omega^{\prime} \rightarrow \Omega$ so that $f$ is not uniformly continuous with respect to the Euclidean metric in $\Omega$ and $d_{I}$ in $\Omega^{\prime}$.

The domain $\Omega$ in Theorem 1.1 cannot be required to be simply connected. More generally, neither the domain in Theorem 1.1 nor in Theorem 1.2 can be required to be quasiconformally equivalent to a uniform domain. For this see [5].

It would be interesting to know whether one could take $s=1+\frac{1}{n-1}$ and dispose with the logarithmic term in Theorem 1.2.

\section{Proofs of the main results}

Proof of Theorem 1.1. Our 2-John domain $\Omega$ will be constructed inductively as indicated in Figure 1.

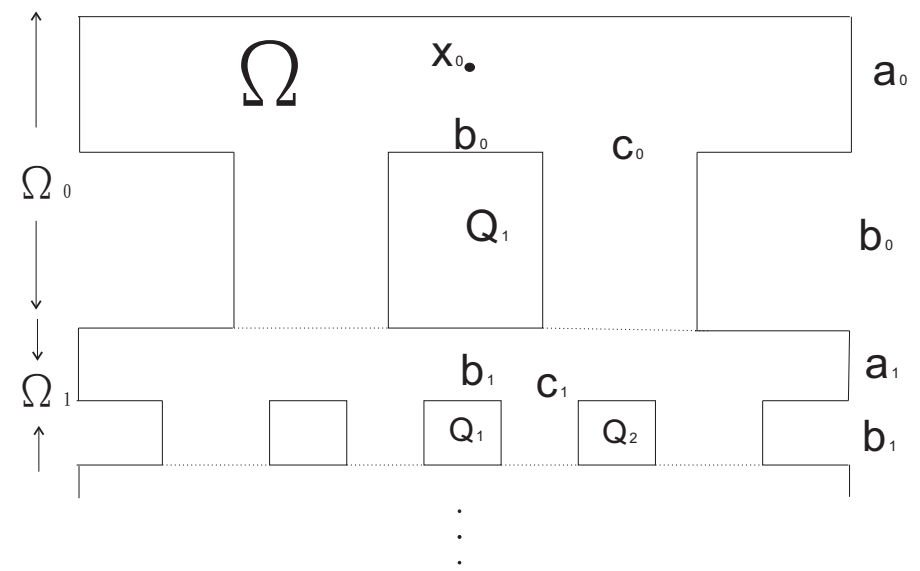

Figure 1. The 2-John domain $\Omega$.

Set $a_{j}=2^{-2(j+1)}, b_{j}=2^{-j}$ and $c_{j}=2^{-2(j+1)}$. For $j=0$, we let the $\Omega_{0}$-part consist of a rectangle of length 1 and width $a_{0}$ centered at the origin, and two rectangular "legs" of width $c_{0}$ and length $b_{0}$. The two rectangular "legs" are obtained in the following manner: first remove the central square $Q_{1}$ of side-length $b_{0}$; then set the distance between $Q_{1}$ and the vertical boundary of $\Omega_{0}$ to be $c_{0}$. Next, for $j=1$, we let the $\Omega_{1}$-part consist of a rectangle of length 1 and width $a_{1}$ and four rectangular "legs" of width $c_{1}$ and length $b_{1}$. The four rectangular "legs" are obtained in a similar fashion as before: first remove 3 squares of side-length $b_{1}$; then make them equi-distributed, i.e. the gap between two consecutive squares is $c_{1}$; finally set the distance between $Q_{2}$ and the vertical boundary of $\Omega_{1}$ to be $c_{1}$. We continue the process. Let the $\Omega_{j}$-part consist of a rectangle of length 1 and width $a_{j}$ and $2^{j}$ rectangular "legs" of width $c_{j}$ and length $b_{j}$. The rectangular "legs" are obtained by removing $2^{j+1}-1$ equi-distributed squares of side-length $b_{j}$ in a similar way as before. Among these removed squares, 
we label from middle to the right-most as $Q_{1}, Q_{2}, \ldots, Q_{2^{j}}$ respectively. According to our construction, the distance between two consecutive removed squares is $c_{j}$ and the distance between $Q_{2^{j}}$ and the vertical boundary of $\Omega_{j}$ is also $c_{j}$. Finally, our domain $\Omega$ is the union of all $\Omega_{j}$ 's. It is clear from the construction that $\Omega$ is 2-John and symmetric with respect to the $y$-axis.

Let $x_{0}=(0,0)$ be the point marked in Figure 1 . It is easy to check that the assumption (1.7) is satisfied.

We next construct our source domain $\Omega^{\prime}$ and a quasiconformal mapping $g: \Omega^{\prime} \rightarrow$ $\Omega$, which is not uniformly continuous with respect to the metrics $d(x, y)=|x-y|$ in $\Omega$ and $d_{I}$ in $\Omega^{\prime}$. Actually, we construct a quasiconformal mapping $f: \Omega \rightarrow \Omega^{\prime}$ whose (quasiconformal) inverse has the desired properties.

The idea is demonstrated in Figure 2: we scale the upper part of each $\Omega_{j}$ by $\frac{1}{j}$ and replace the associated $2^{j+1}$ rectangular "legs" by the same number of new "legs". The vertical distance between the scaled upper parts of $\Omega_{j}$ and $\Omega_{j+1}$ is set to be $2 j^{-2}$. We also make the domain $\Omega^{\prime}$ symmetric with respect to $y$-axis. Since the distance between two consecutive legs in $\Omega_{j}$ is $2^{-j}$, the distance between the tops of two consecutive "legs" in $\Omega_{j}^{\prime}$ is $\frac{2^{-j}}{j}$. For the bottoms, the distance is approximately $\frac{2^{-j-1}}{j+1}$.

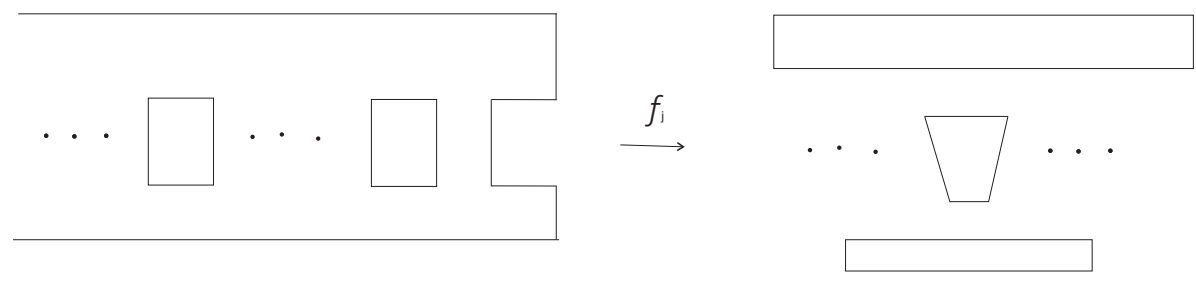

Figure 2. $\Omega$ and $\Omega^{\prime}$ in the step $j$.

Recall the labelled squares $Q_{i}, i=1, \ldots, 2^{j}$ introduced in $\Omega_{j}$. We denote by $\tilde{Q}_{i}$ the "leg" next to $Q_{i}$, on the right. We will construct a quasiconformal mapping $f_{j}$ from the (translated) rectangle $\tilde{Q}_{i}$ to the (translated) new "leg" $Q_{i}^{\prime}$ as in Figure 3. $Q_{i}^{\prime}$ consists of two parts $A^{\prime}$ and $B^{\prime}$. The distance between the bottom line segment $0 \mathbf{a}$ and the top line segment in the $x$-direction is

$$
m_{i}^{j}=\frac{\left[2^{-j-1}+2^{-2(j+1)}\right] \cdot i}{j}-\frac{\left[2^{-j-1}+2^{-2(j+1)}\right] \cdot i}{j+1} .
$$

It is clear that $m_{i}^{j} \approx \frac{i \cdot 2^{-j}}{j^{2}}$ when $j$ is large. The distance of the top and the bottom in $y$-direction is $\frac{2}{j^{2}}$. In Figure $3, \mathbf{a}=\left(\frac{2^{-2(j+1)}}{j+1}, 0\right), \mathbf{p}=\left(\frac{i \cdot 2^{-j}}{2 j^{2}}, \frac{1}{j^{2}}\right)$ and $\mathbf{q}=$ $\left(\frac{i \cdot 2^{-j}}{2 j^{2}}+\frac{2^{-(j+1)}}{j}, \frac{1}{j^{2}}\right)$. We will write down below a quasiconformal mapping $f_{j}: A \rightarrow A^{\prime}$ such that $f_{j}$ maps the bottom line segment of $A$ linearly to $0 \mathbf{a}$ and the top line segment of $A$ affinely to $\mathbf{p q}$, respectively. The line $0 \mathbf{p}$ is of the form $y=k_{1} x$, where

$$
k_{1}=\frac{1 / j^{2}}{i \cdot 2^{-j} /\left(2 j^{2}\right)}=\frac{2^{j+1}}{i} \geq 1 \text {. }
$$

Similarly, the line aq is of the form $y=k_{2}\left(x-\frac{2^{-2(j+1)}}{j+1}\right)$, where

$$
k_{2}=\frac{1 / j^{2}}{\frac{i \cdot 2^{-j}}{2 j^{2}}+\frac{2^{-(j+1)}}{j}-\frac{2^{-2(j+1)}}{j+1}} \approx \frac{2^{j}}{i+j} .
$$




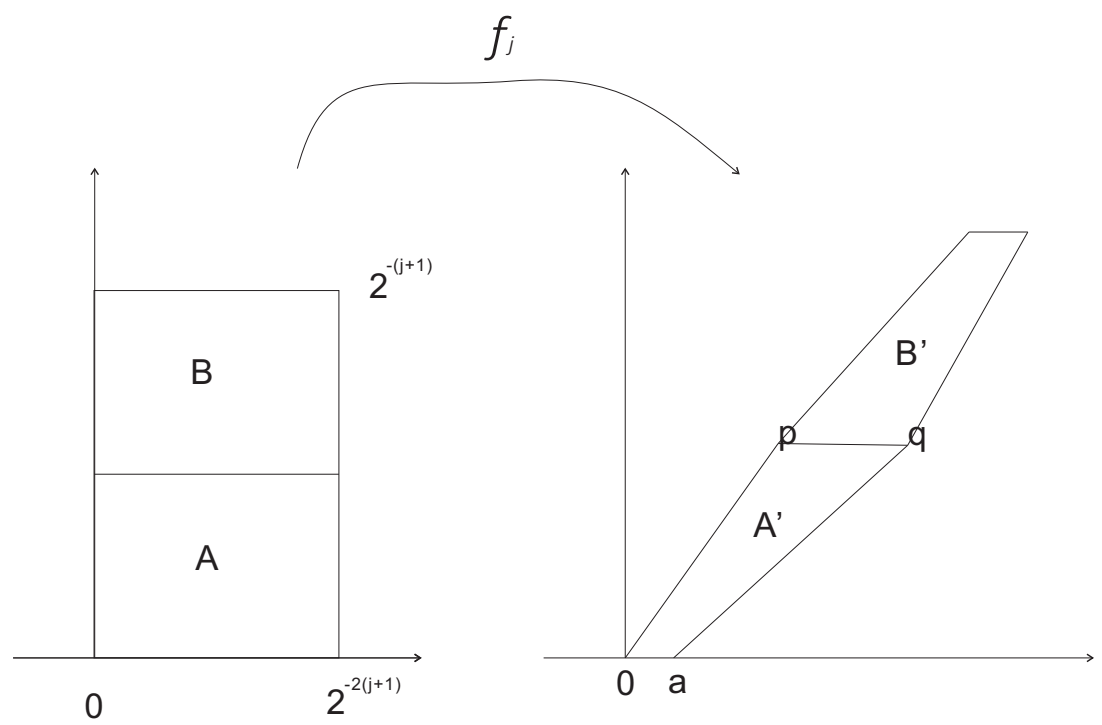

Figure 3. The quasiconformal mapping from $\tilde{Q}_{i}$ to $Q_{i}^{\prime}$.

We are looking for a quasiconformal mapping of the form $f_{j}^{i}(x, y)=\left(\tilde{g}_{j}(y) x+\right.$ $\left.g_{j}(y), k_{1} g_{j}(y)\right)$, where $\tilde{g}_{j}(y)=k_{1} g_{j}^{\prime}(y)$ for all $y \in\left[0,2^{-(j+1)}\right]$ and $g_{j}$ is a smooth increasing function. Clearly, such a mapping $f_{j}$ maps horizontal line segments to horizontal line segments. We further require that it maps the left side of $A$ to $0 \mathbf{p}$ and the right side of $A$ to aq, $g_{j}(0)=0, g_{j}\left(2^{-j}\right)=\frac{1}{j^{2}}$ and $\tilde{g}_{j}(0)=\frac{1}{j+1}$. By definition,

$$
f_{j}^{i}\left(2^{-2(j+1)}, y\right)=\left(\tilde{g}_{j}(y) \cdot 2^{-2(j+1)}+g_{j}(y), k_{1} g_{j}(y)\right) .
$$

The further requirements are satisfied if $\tilde{g}_{j}=k_{1} g_{j}^{\prime}$,

$$
\begin{gathered}
g_{j}(y)=k_{2} \cdot k_{1}^{-1} \tilde{g}_{j}(y) \cdot 2^{-2(j+1)}+\frac{k_{2}}{k_{1}} g_{j}(y)-\frac{k_{2}}{j} \cdot 2^{-2(j+1)}, \\
g_{j}(0)=0, g_{j}\left(2^{-j}\right)=\frac{1}{j^{2}} \quad \text { and } \quad \tilde{g}_{j}(0)=\frac{1}{j+1} .
\end{gathered}
$$

One can easily solve the above system of equations by setting $g_{j}(y)=a \cdot e^{a_{j}^{i} y+c}-b$, where

$$
a_{i}^{j}=2^{2(j+1)} \frac{k_{1}-k_{2}}{k_{1} k_{2}}, \quad b=\frac{1}{k_{1}(j+1) a_{j}^{i}}
$$

and the constants $b$ and $c$ are chosen such that

$$
a \cdot e^{c}=b \quad \text { and } \quad a \cdot e^{a_{j}^{i} 2^{-j}+c}-b=\frac{1}{j^{2}} .
$$

We next show that $f_{j}^{i}$ is a quasiconformal mapping. A direct computation gives us

$$
D f_{j}^{i}(x, y)=\left[\begin{array}{cc}
\tilde{g}_{j}(y) & \tilde{g}_{j}^{\prime}(y) x+g_{j}^{\prime}(y) \\
0 & k_{1} g_{j}^{\prime}(y)
\end{array}\right]
$$

We only need to show that $\tilde{g}_{j}^{\prime}(y) x+g_{j}^{\prime}(y) \leq M k_{1} g_{j}^{\prime}(y)$, for some constant $M$ independent of $i$ and $j$, and for all $x, y \in A$. Since $k_{1} \geq 1$, it suffices to bound $\tilde{g}_{j}^{\prime}(y) x$. By definition,

$$
\tilde{g}_{j}(y)=k_{1} g_{j}^{\prime}(y)=k_{1} a a_{j}^{i} e^{a_{j}^{i} y+c}
$$


and

$$
\tilde{g}_{j}^{\prime}(y)=k_{1} a_{j}^{i} g_{j}^{\prime}(y)
$$

Hence we only need to find a uniform bound on $x \cdot a_{j}^{i}$. For this, we first note that $k_{2}$ is bounded from below by $\frac{1}{2}$ and $\frac{k_{1}-k_{2}}{k_{1}} \leq 1$. Since $x \in\left[0,2^{-2(j+1)}\right]$, we have

$$
a_{j}^{i} x \leq \frac{k_{1}-k_{2}}{k_{1} k_{2}} \cdot 2^{2(j+1)} x \leq 2 .
$$

This implies that $\tilde{g}_{j}^{\prime}(y) x+g_{j}^{\prime}(y) \leq 3 k_{1} g_{j}^{\prime}(y)$ and so $f_{j}^{i}$ is quasiconformal. Notice that $f_{j}^{i}(x, 0)=\left(\frac{x}{j+1}, 0\right)$, so that, after suitable translations, $f_{j}^{i}$ matches with our scaling on the top of $\Omega_{j+1}$. In a similar manner, one can write down a quasiconformal mapping from $B$ to $B^{\prime}$ such that it coincides with $f_{j}^{i}$ on pq and is linear on each line segment. In fact, the quasiconformal mapping just slightly differs from the reflection of $f_{j}$ with respect to the line segment pq (since the length of $0 \mathbf{a}$ is approximately the same as the length of the top line segment when $j \rightarrow \infty$ and the picture is exactly a reflection with respect to pq). When a suitable coordinate system is fixed, it is clear that the mappings $f_{j}^{i_{1}}$ and $f_{j}^{i_{2}}$ only differ by a translation in $x$-direction and hence the desired global quasiconformal mapping $f_{j}$ from $\Omega_{j}$ to $\Omega_{j}^{\prime}$ follows by gluing all $f_{j}^{i}$ 's and the scaling maps.

In this manner, the domain $\Omega^{\prime}$ is well-defined. We can define the quasiconformal mapping $g: \Omega^{\prime} \rightarrow \Omega$ by setting $\left.g\right|_{\Omega_{j}^{\prime}}=f_{j}^{-1}$. Moreover, $g$ cannot be uniformly continuous since for each $j \in \mathbf{N}$, it maps a rectangle of length $\frac{1}{j}$ linearly to a rectangle of length 1.

Proof of Theorem 1.2. We will give the detailed constructions of our domains and quasiconformal mapping for $n=3$ and indicate how to pass them to all dimensions at the end of the proof. The idea of the 3-dimensional construction is similar to the one above and we simply fatten the " $\Omega_{0}$ " part of the planar domain in Figure 1 along the third direction; see Figure 4 below.

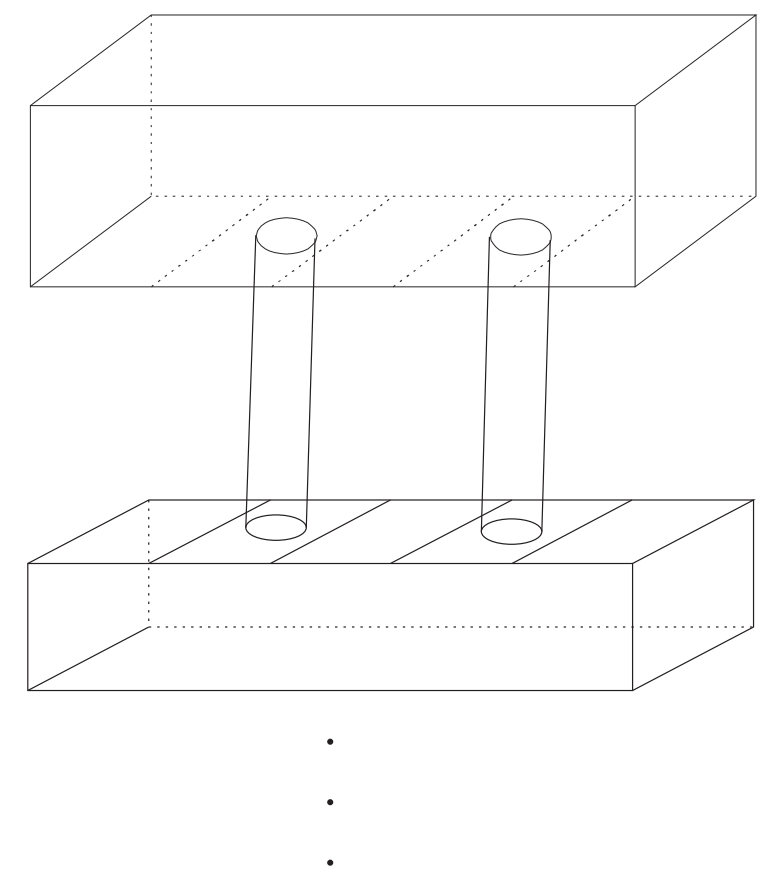

Figure 4. The first part of our domain $\Omega$. 
The top part of Figure 4 consists of a rectangle of length 1, width $\frac{1}{2^{2}}$ and height $\frac{1}{2^{3}}$. In the bottom, the rectangle has length 1 , width $\frac{1}{2^{4}}$ and height $\frac{1}{2^{6}}$. We attach four cylindrical "legs" of height $2 \cdot 2^{-2}$ between these rectangles. The radius of the cylinder is about $2^{-3}$ and the distance between them is about $2^{-2}$.

We can proceed our construction in the following manner. At step $j$, the top part consists of a rectangle of length 1 , width $2^{-2 j}$ and height $2^{-3 j}$. In the bottom, the rectangle has length 1 , width $2^{-2(j+1)}$ and height $2^{-3(j+1)}$. We attach $2^{2 j}$ equidistributed cylindrical "legs" of height $2^{-2 j}$ between them. The radius of the cylinder is about $2^{-3 j}$ and the distance between two consecutive cylinders is about $h_{j}=j \cdot 2^{-2 j}$. It is clear from our construction that $\Omega$ is an $s$-John domain for any $s \in\left(1+\frac{1}{2}, \infty\right)$.

Let $x_{0}$ be the central point in the first rectangle of $\Omega$. It is easy to check that the assumption (1.8) is satisfied.

Our source domain $\Omega^{\prime}$ is obtained by a similar scaling procedure as in the proof of Theorem 1.1. To be more precise, at step $j$, we scale the top rectangle by $\frac{1}{j^{2}}$ and replace the associated $2^{j}$ cylindrical "legs" by the same number of new "legs". The vertical distance between the scaled top rectangle and the bottom rectangle is set to be $h_{j}^{\prime}=\frac{2}{j^{2}}$.

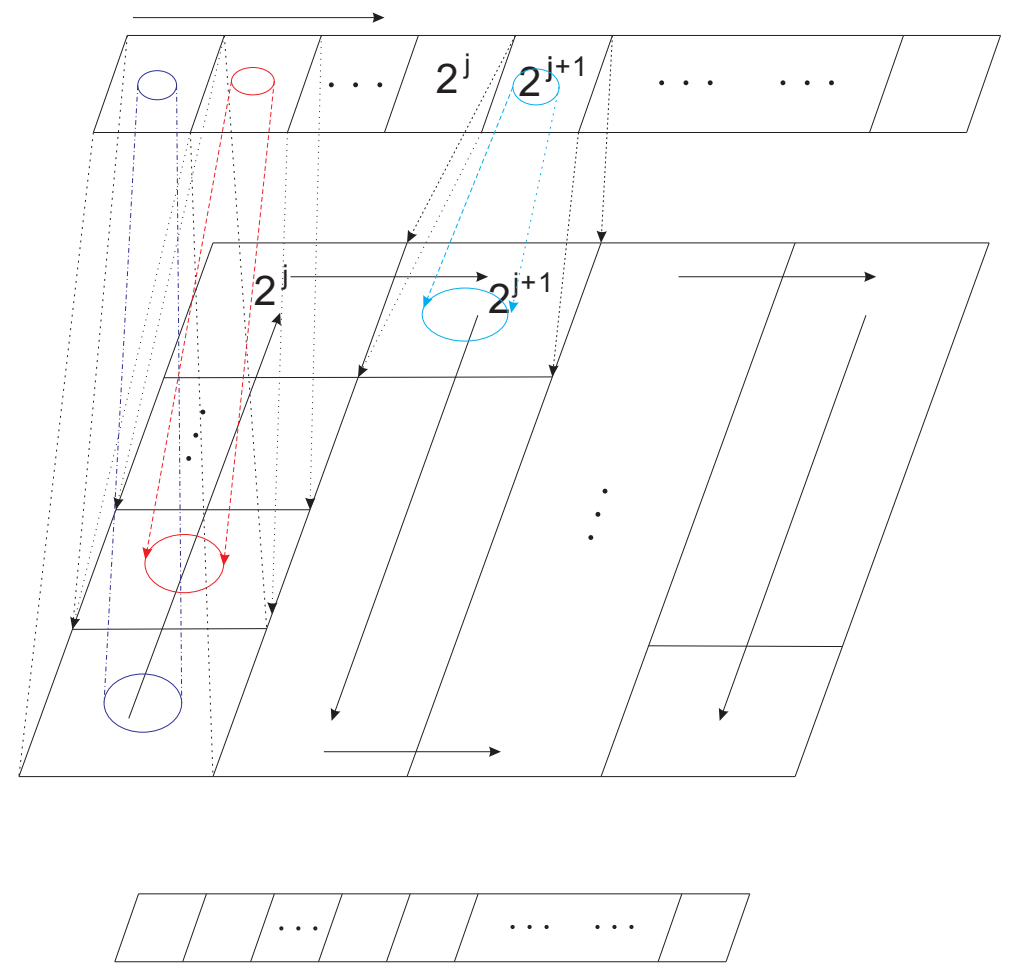

Figure 5. The new "legs" at step $j$.

We next explain how to select the new "legs", see Figure 5 for a top view. In Figure 5, the top rectangle has length $\frac{1}{j^{2}}$ and width $\frac{2^{-2 j}}{j^{2}}$. It consists of $2^{2 j}$ squares of side-length $\frac{2^{-2 j}}{j^{2}}$. The bottom rectangle has length $\frac{1}{(j+1)^{2}}$ and width $\frac{2^{-2(j+1)}}{(j+1)^{2}}$. The vertical distance between these rectangles is $h_{j}^{\prime}$. We insert a square $S_{j}$ of side-length $\frac{1}{j^{2}}$ in the middle of the two rectangles, i.e. the (vertical) distance between $S_{j}$ and either of the rectangles is $\frac{1}{j^{2}}$. We divide $S_{j}$ into $2^{2 j}$ subsquares of side-length $\frac{2^{-j}}{j^{2}}$. Next, we set up a one-to-one correspondence between the $2^{2 j}$ squares in the top rectangle and the subsquares in $S_{j}$. To be more precise, we first construct $2^{2 j}$ affine 
"rectangles" between each square in the top rectangle and each subsquare in $S_{j}$ and then we insert a "cylindrical leg" inside each affine "rectangle", see Figure 5 for the order of the affine "rectangles". The radius of the top circle of the "cylindrical leg" is set to be $\frac{2^{-3 j}}{j^{2}}$ and the radius of the bottom circle is $\frac{2^{-j}}{j^{2}}$. Since the $2^{2 j}$ affine "rectangles" have disjoint interiors, the $2^{2 j}$ "cylindrical legs" are pairwise disjoint. As in the proof of Theorem 1.1, we use a similar construction between $S_{j}$ and the bottom rectangle.

Reasoning as in the proof of Theorem 1.1, we only need to write down quasiconformal mappings between these "legs". Note that our construction implies that all the $2^{2 j}$ "cylindrical legs" are bi-Lipschitz equivalent, with a constant independent of $j$. So finally we reduce the problem to the existence of a quasiconformal mapping $g$ as in Figure 6.
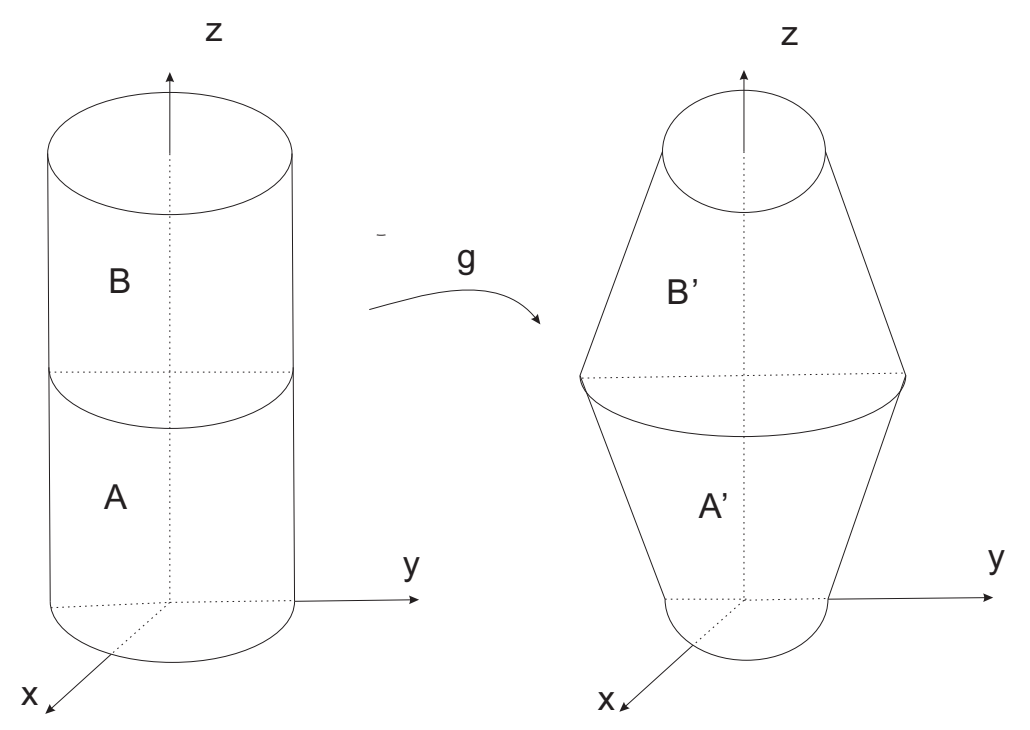

Figure 6. The quasiconformal mapping from a "cylinder" to a "double cone".

We will use the coordinate system marked in Figure 6 and write down a quasiconformal mapping $g$ from $A$ onto $A^{\prime}$ such that $g$ is a scaling between the bottom and top disks. Set

$$
g(x, y, z)=\left(g_{1}(z) x, g_{1}(z) y, g_{2}(z)\right) .
$$

We require that $g_{1}(0)=\frac{1}{j^{2}}, g_{1}\left(h_{j}\right)=\frac{2^{2 j}}{j^{2}}, g_{2}(0)=0, g_{2}\left(h_{j}\right)=h_{j}^{\prime}$ and $g_{2}^{\prime}(z)=g_{1}(z)$ for all $z \in\left[0, h_{j}\right]$. It is easy to check that with these requirements, $g$ will be a quasiconformal mapping that maps $A$ to $A^{\prime}$ such that $g$ is the desired scaling between the bottom and top disks. One can use a map $g_{2}$ of the form $g_{2}(z)=a_{j}\left(e^{b_{j} z}-1\right)$, where $a_{j} \approx \frac{2^{-2 j}}{j^{2}}$ and $b_{j} \approx 2^{2 j}$.

As in the planar case, the global quasiconformal mapping $f: \Omega^{\prime} \rightarrow \Omega$ is obtained by gluing all these $g^{\prime}$ s and the corresponding scaling mappings. Moreover, reasoning as in the planar case, we can easily conclude that $f$ cannot be uniformly continuous with respect to the metrics $d(x, y)=|x-y|$ in $\Omega$ and $d_{I}$ in $\Omega^{\prime}$.

The construction of the general $n$-dimensional case can be proceeded in a similar manner. At step $j, \Omega_{j}$ consists of a $n$-dimensional rectangle of length $a_{1}=1$ and (other) edge-lengths $a_{2}=\cdots=a_{n-1}=2^{-(n-1) j}, a_{n}=2^{-n j}$ and $2^{j}$ "cylindrical legs" of length $h_{j}=j \cdot 2^{-(n-1) j}$. The radius of the cylinder is $2^{-n j}$. So $\Omega$ is an $s$-John domain for any $s \in\left(1+\frac{1}{n-1}, \infty\right)$. 
The source domain $\Omega^{\prime}$ is obtained by a similar scaling procedure as before. To be more precise, at step $j$, we scale the top rectangle by $\frac{1}{j^{2}}$ and replace the associated $2^{j}$ cylindrical "legs" by the same number of new "legs". The vertical distance between the scaled top rectangle and the bottom rectangle is set to be $h_{j}^{\prime}=\frac{2}{j^{2}}$.

We use a similar idea as before to obtain new "legs" between the top rectangle and bottom rectangle as in Figure 5. Namely, we insert a $(n-1)$-dimensional cube of edge-length $\frac{1}{j^{2}}$ and then divide it into $2^{(n-1) j}$ subcubes of edge-length $\frac{2^{-j}}{j^{2}}$. Then attach $2^{(n-1) j}$ affine "rectangles" in a similar manner as before. Inside each affine "rectangle", we insert a "cylindrical leg". The radius of the top of the "cylindrical leg" is $\frac{2^{-n j}}{j^{2}}$ and the radius of the bottom is $\frac{2^{-j}}{j^{2}}$. Reasoning as before, one essentially only needs to write down a quasiconformal mapping $g$ between these "legs".

The global quasiconformal mapping $f: \Omega^{\prime} \rightarrow \Omega$ is obtained by gluing all these $g^{\prime}$ s and the corresponding scaling mappings. Moreover, reasoning as in the planar case, we can easily conclude that $f$ cannot be uniformly continuous with with respect to the metrics $d(x, y)=|x-y|$ in $\Omega$ and $d_{I}$ in $\Omega^{\prime}$.

\section{References}

[1] Astala, K., T. Iwaniec, and G. Martin: Elliptic partial differential equations and quasiconformal mappings in the plane. - Princeton Univ. Press, Princeton, NJ, 2009.

[2] Becker, J., and Ch. Pommerenke: Hölder continuity of conformal maps with quasiconformal extension. - Complex Var. Theory Appl. 10:4, 1988, 267-272.

[3] Gehring, F. W., and O. Martio: Lipschitz classes and quasiconformal mappings. - Ann. Acad. Sci. Fenn. Ser. A I Math. 10, 1985, 203-219.

[4] Gehring, F. W., and B. G. Osgood: Uniform domains and the quasihyperbolic metric. - J. Anal. Math. 36, 1979, 50-74.

[5] Guo, C.-Y.: Uniform continuity of quasiconformal mappings onto generalized John domains. - Ann. Acad. Sci. Fenn. Math. 40:1, 2015, 183-202.

[6] Guo, C.-Y.: Generalized quasidisks and conformality II. - Proc. Amer. Math. Soc. 143:8, $2015,3505-3517$.

[7] Guo, C.-Y., P. Koskela, and J. Takkinen: Generalized quasidisks and conformality. Publ. Mat. 58:1, 2014, 193-212.

[8] Hencl, S., and P. Koskela: Quasihyperbolic boundary conditions and capacity: uniform continuity of quasiconformal mappings. - J. Anal. Math. 96, 2005, 19-35.

[9] John, F.: Rotation and strain. - Comm. Pure Appl. Math. 14, 1961, 391-413.

[10] Koskela, P., J. Onninen, and J.T. Tyson: Quasihyperbolic boundary conditions and capacity: Hölder continuity of quasiconformal mappings. - Comment. Math. Helv. 76:3, 2001, $416-435$.

[11] Martio, O., and J. Sarvas: Injectivity theorems in plane and space. - Ann. Acad. Sci. Fenn. Ser. A I Math. 4:2, 1979, 383-401.

[12] NÄKKI, R., and J. VÄISÄLÄ: John disks. - Expo. Math. 9:1, 1991, 3-43.

[13] Smith, W., and D. A. Stegenga: Hölder and Poincaré domains. - Trans. Amer. Math. Soc. 319, 1990, 67-100. 